\title{
Die Abweichung vom Exponentialzerfall angeregter Zustände
}

\author{
Ketill Ingólfisson \\ Mathematisches Institut der Universität Zürich * \\ Eingegangen am 28. Mai 1968
}

\begin{abstract}
In the theory of line breadth phenomena excited amplitudes must not exactly follow the usual exponential decay law. This fact is well known from fundamental mathematical considerations, although there is not any unique form for the corresponding deviation. In this paper we seek a new expression for the decay, based upon a rather general field model of mass zero. The result is a good approximation for times $\sim 1 / \gamma$ and yields the exact asymptotic solution. A modification to a model with mass $\neq 0$ does not in general lead to other formulations of the solution.
\end{abstract}

\section{$\$ 1$. Einfïhrung}

In der Elementarteilchenphysik und in der Strahlungstheorie werden spontane Zerfallsprozesse meistens so angefaßt, daß zuerst der angeregte Zustand $\Psi$ zu einer endlichen Zeit in konkreter Form angegeben wird. Nachdem der darauffolgende Zerfall die Zeit $t$ gedauert hat, ist dann laut den Grundprinzipien der Quantentheorie der Zustand in der Form

$$
\Psi_{t}=e^{-i H t} \Psi \text {. }
$$

Der Ausdruck (1) ist hier nicht nur rein formal, sondern als das Ergebnis der modernen Spektraltheorie im Sinne J. v. NEumanns [1] zu verstehen. Es fragt sich aber, ob der exponentielle Charakter der Gesamtlösung zu einer reellen Exponentialabhängigkeit führen kann, wenn die einzelnen Matrixelemente berechnet werden. Die klassische Quantentheorie [2] setzt bei Zerfallsprozessen die Lösung der Feldgleichungen in der Form einer Exponentialfunktion an. Dadurch erhält man eine exponentielle Abnahme der Zerfallsamplituden. Das Verfahren wird durch die experimentelle Untersuchung vieler verschiedener Zerfallsmechanismen ausgezeichnet bestätigt. Trotzdem besteht zwischen der Form (1) und diesem Resultat ein Widerspruch: Die klassische Quantentheorie betrachtet den Zustand $\Psi$ als eine beliebige Überlagerung von Eigenzuständen des $H$-Operators. Da dieser aber selbstadjungiert ist, kann im Allgemeinen keine rein exponentielle Abnahme in (1) bestehen. Die gleiche Situation entsteht in jeder neueren Theorie, die den Anfangszustand im gleichen Sinne betrachtet. Der theoretischen Begründung der Exponentialabnahme geht deshalb gewöhnlich die Untersuchung voraus, ob sie als eine gute Näherung gelten darf. Die erste Arbeit dieser Art leisteten WEIssKopF und WIGNer [3] in der Frage der spontanen Lichtemission. Die genauesten

\footnotetext{
* Jetzt: Dept. of Mathematics, University of Arizona.
} 
Resultate stammen aber von W. HeITLer und seinen Schülern [4-8], die das Problem in ihrer allgemeinen Dämpfungstheorie diskutiert haben.

Über die wirkliche Form der Abweichung der exakten Lösung vom Exponentialzerfall sind bis jetzt wenige Arbeiten erschienen. In ihrer ausführlichen Analyse der relativistischen Theorie instabiler Teilchen schätzten Matthews und SaLAM [9] die Abweichung bei $\pi$-Zerfall als eine Störung ab, die erst in Erscheinung tritt, wenn die Zeitspanne nach ihrer Erzeugung etwa die 100 malige Lebensdauer erreicht hat. Nach ihrer Aussage aus privater Quelle hat M. LÉvy in seinen Untersuchungen des Lee-Modells als erster erkannt, daß die Abweichung vom Exponentialzerfall in negativen Potenzen der Zeit bestehen könne. Die erste allgemeine Formulierung der Abweichung als Integral um einen Verzweigungspunkt am Anfang des Streckenspektrums stammt von G. HöHLER [10]. Er geht auf eine Idee von HeLLund [11] zurück, der die Grundgleichungen Laplacetransformiert hat. Konkrete Resultate hat HöHLER im speziellen Fall des Lee-Modells bei besonderer Annahme über die Abschneidefunktion erreicht. Seine Lösung ist eine asymptotische Reihe in $t^{-(n+1 / 2)}$. Das erste Glied der Reihe ist proportional $t^{-3 / 2}$. Allgemeingültige Formen der Laplacetransformierten Grundgleichungen finden wir bei G. KäLlén [12] und A. Messiah [13]. Die analytische Fortsetzung von den Integranden der Zurücktransformation finden wir aber erst bei M. FRIEDRICH [14], die jedoch nur ein einfaches Modell der Quantenelektrodynamik anwendet. Die konkrete Auswertung des Integrals um den Verzweigungspunkt hat bis jetzt gefehlt. Es ist das Ziel der vorliegenden Arbeit, eine derartige Auswertung unter allgemeingültigen Voraussetzungen auszuführen.

Wir können die Theorie der spontanen Zerfallsprozesse von einem allgemeinen Standpunkt aus betrachten, ohne zuerst die Menge der Eigenzustände oder das Spektrum des $H$-Operators kennenzulernen. $\mathrm{Zu}$ nächst kommen wir dann zu einer einfachen Forderung über den Anfangszustand. Wir gehen von der Gl. (1) aus und betrachten die Lösung $\Psi_{t}$ als einen Vektor im allgemeinen Hilbertraum $R$. Es sei $g$ eine komplexe Zahl mit dem Realteil $\xi$. Wir bilden die Laplacetransformierte $\bar{\Psi}(g)$ des Zustandsvektors und erhalten die Gleichung

$$
(g+i H) \bar{\Psi}(g)=\Psi, \quad \xi>0 .
$$

Dann bilden wir die Zurücktransformierte im Sinne der Laplacetransformation:

$$
\Psi(t)=\frac{1}{2 \pi i} \int_{\xi-i \infty}^{\xi+i \infty} e^{g t} \bar{\Psi}(g) d g, \quad \xi>0 .
$$

Der Verfasser hat in einer früheren Arbeit über die Theorie der natürlichen Linienbreite [15] den folgenden Satz bewiesen:

Wenn $\Psi$ ein Element des Hilbertraumes $R$ ist, gibt es immer eine Lösung der Laplacetransformierten Wellengleichung (2). Wenn $\Psi$ dem 
Definitionsbereich $D(H)$ des Operators $H$ angehört, so ist durch die Umkehrtransformation (3) ein Zustand $\Psi(t)$ definiert, der die zeitabhängige Schrödingergleichung erfüllt und für $t \rightarrow 0$ gegen $\Psi$ strebt. Es ist dann $\Psi_{t} \equiv \Psi(t)$.

Damit ist die Grundlage einer Lösungsmethode gegeben, die das betreffende physikalische Problem zuerst in seiner Laplacetransformierten Gestalt löst. Wenn eine Realisation des Hilbertschen Raumes gegeben ist und die Lösung $\Psi(g)$ der Gl. (2) bekannt ist, nennen wir das Integral (3) die formale Lösung des Problems.

Jede bestimmte Form der Quantentheorie setzt noch die Existenz des Operators der freien Energie $H_{0}$ voraus, der auch selbstadjungiert ist. Man kann dann verschiedene Modelle von dem Standpunkt $\Psi \in D\left(H_{0}\right)$ aus im allgemeinen Raum diskutieren ${ }^{1}$. Im folgenden wollen wir jedoch eine bestimmte Raumstruktur anwenden und auf die Darstellung nach Teilchenzahl zurückgreifen, die in [15] nach den bekannten Ideen von V. Fock [16], L. Schwartz [17], K. Friedrichs [18] und B. L. van DER WAERDEN [19] diskutiert wurde. Der Operator $H_{0}$ soll in bezug auf diese Struktur diagonal sein und die physikalisch gut begründete Forderung

$$
D(H)=D\left(H_{0}\right) \leqq D\left(H-H_{0}\right)=R
$$

erfüllen. Die Darstellung soll eine so kleine Teilchenzahl berücksichtigen, daß die formale Lösung ohne Aufsummierung einer unendlichen Reihe gleich zu Beginn aufgestellt werden kann. Diese Forderungen wurden in einem physikalischen Beispiel erfüllt, das am Schluß der Arbeit [15] diskutiert wurde: Die spontane Lichtemission formuliert in einem Hilbertraum, der außer den Bestimmungsgrößen des Elektrons nur diejenigen eines Photons berücksichtigt. Die formale Lösung kann in diesem Hilbertraum minimalster Teilchenzahl für viele andere Modelle aus der Theorie der Elementarteilchen stellvertretend sein. Wir wollen sie deshalb als Modell allgemein formulieren.

Im folgenden werten wir zwei Integrale aus, die als Verallgemeinerungen der in [15] begründeten formalen Lösung gelten können. In dieser Form scheinen sie uns typisch für die Zerfallsprozesse der Quantentheorie. Wir studieren insbesondere dieArt der Abweichung vom Exponentialzerfall.

Es ist die Ansicht des Verfassers, daß der Übergang zum Hilbertraum unbeschränkter Teilchenzahl auf eine Renormierung der formalen Lösung im ,kleinen“ Raum zurückgeführt werden kann. Im Rahmen einer allgemeineren Theorie kommt der vorliegenden Auswertung somit erhöhte Bedeutung zu. Grundsätzliche physikalische Einwände gegen diese Arbeit sind durch Zweifel an der Brauchbarkeit des Fockraumes gegeben, sowie durch die weitverbreitete Meinung, daß ein Anfangszustand $\Psi$ nicht fixiert werden kann. Es soll hier nicht gegen solche Einwände Stellung genommen werden.

\footnotetext{
1 Eine weitere Arbeit des Verfassers behandelt dieses Thema.
} 


\section{§. Die formale Lösung}

Es sei ein physikalisches Problem gegeben, das durch die beiden folgenden Wahrscheinlichkeitsamplituden beschrieben wird:

$$
\begin{aligned}
\psi_{1}(t) & =\frac{1}{2 \pi i} e^{-i \omega_{2} t} \int_{\xi-i \infty}^{\xi+i \infty} e^{z t} \frac{1}{z+i \omega_{0}+\Gamma(z)} d z, \\
\psi_{2}(k, r, t) & =\frac{1}{2 \pi i} e^{-i \omega_{2} t} \int_{\xi-i \infty}^{\xi+i \infty} e^{z t} \frac{\left\{i h_{i}(1 / 2, k, r)\right\}^{*}}{(z+i|k|)\left(z+i \omega_{0}+\Gamma(z)\right)} d z .
\end{aligned}
$$

Bei der Integration soll $\xi>0$ sein. Die Funktion $\Gamma(z)$ sei durch das Integral

$$
\Gamma(z)=\int \sum_{r=1}^{3} \frac{d^{3} k}{2|k|} \frac{\left|\varphi_{r}(k)\right|^{2}}{z+i|k|}
$$

gegeben. Die beiden Grenzwerte

$$
\begin{aligned}
\frac{\gamma}{2} & =\operatorname{Re} \lim _{\xi \rightarrow+0} \Gamma\left(\xi-i \omega_{0}\right), \\
\triangle \omega_{0} & =\operatorname{Im} \lim _{\xi \rightarrow+0} \Gamma\left(\xi-i \omega_{0}\right)
\end{aligned}
$$

seien endlich und ihre Beträge sehr klein gegenüber $\omega_{0}=\omega_{1}-\omega_{2}$, der Differenz der Energiewerte des angeregten und des zerfallenen Teilchens.

Die Zahlen $\omega_{0}, \gamma$ und $\triangle \omega_{0}$ bilden die wichtigsten Konstanten in der Theorie der natürlichen Linienbreite. Die obigen Angaben können aber dann erst ein brauchbares Modell für einen Zerfallsprozeß bilden, wenn zusätzliche Eigenschaften der drei Funktionen $\varphi_{r}(k)(r=1,2,3)$ gegeben sind. Wir setzen somit voraus:

a) Für jedes $r$ besitze $\left|\varphi_{r}(k)\right|$ ein endliches Quadratintegral.

b) Esgebe einen endlichen Grenzwert vonjeder $\varphi_{r}(k)$ wenn $|k| \rightarrow 0$ strebt. Wegen a) ist es klar, daß die Fouriertransformierten

$$
\varphi_{r}(x)=(2 \pi)^{-3 / 2} \int d^{3} k e^{-i k x} \varphi_{r}(k)
$$

existieren und daß jedes $\left|\varphi_{r}(x)\right|$ das gleiche Quadratintegral besitzt wie $\left|\varphi_{r}(k)\right|$. Wir definieren $f$ als eine reelle positive $\mathrm{Zahl}$ so daß

$$
f^{2}=\sum_{r}\left|\varphi_{r}(k=0)\right|^{2} \cdot(2 \pi)^{3}
$$

ist und sehen daß folgendes wegen b) behauptet werden darf:

$$
f^{2}=\iint \sum_{r} \varphi_{r}(x) \varphi_{r}^{*}\left(x^{\prime}\right) d^{3} x d^{3} x^{\prime} .
$$

Die Funktion $f^{-2} \sum_{r} \varphi_{r}(x) \varphi_{r}^{*}\left(x^{\prime}\right)$ wenden wir im folgenden als eine Verteilungsfunktion im $\left\{x, x^{\prime}\right\}$-Raum an und bezeichnen die entsprechenden Erwartungswerte mit einem oberen Querstrich. Wir können dann die zwei letzten Voraussetzungen so formulieren:

c) Die reelle Zahl $\overline{\left|x-x^{\prime}\right|^{n}}$ sei endlich und positiv für jedes $n$.

d) Die reelle Zahl $\overline{e^{\left|x-x^{\prime}\right|} \xi}$ sei $<f^{-2}$ für jedes $0 \leqq \xi \leqq \gamma$. 
Wir sehen, daß die Formeln (5) und (6) bis auf Vereinfachungen der Schreibweise mit den Formeln (3) und (4) S. 257 in [15] in der Theorie der Lichtemission übereinstimmen. Ebenfalls ist die Form der $\Gamma$-Funktion nach (7) samt den Voraussetzungen a), b) und c) für die $\varphi$-Funktionen im gleichen physikalischen Beispiel erfüllt. (Siehe in [15] die Formeln (23) S. 256 und (19) S. 261.) In Anlehnung an die Quantenelektrodynamik behalten wir den Index $r$ (Polarisation des Lichtes), aber sein Weglassen tut sonst in der folgenden Diskussion nichts zur Sache.

Hätten wir die in der Einführung formulierte Lösungsmethode zur Herleitung der Integrale (5) und (6) in einem konkreten physikalischen Fall gebraucht, so wären sie dann und nur dann sinnvoll und die richtigen Lösungen der ursprünglichen Wellengleichung, wenn das Integral

$$
\int \sum_{r} \frac{d^{3} k}{2|k|}\left|\varphi_{r}(k)\right|^{2}
$$

konvergiert. Diese Bedingung folgt aus dem Satz, der in der Einleitung zitiert wird, wenn der Anfangszustand als voll angeregt betrachtet wird. (Siehe [15] S. 252.) Die Bedingung ist für das gegebene Verhalten der $\varphi$-Funktionen erfüllt. (Siehe [15] S. 256.) Es folgt nun auch, daß $\Gamma(z)$ für alle $z$, die nicht auf der negativen imaginären Achse liegen, konvergiert und eine analytische Funktion von $z$ darstellt. (Siehe [15] S. 257.) Der Nullpunkt ist ein Verzweigungspunkt und wir können uns die $z$-Ebene längs der negativen imaginären Achse aufgeschnitten denken. Wenn wir an die Ufer des Schnittes gehen, ist

$$
\begin{gathered}
\lim _{\substack{\xi \rightarrow+0 \\
\eta<0}} \Gamma(\xi+i \eta)=-\left\{\lim _{\substack{\xi \rightarrow-0 \\
\eta<0}} \Gamma(\xi+i \eta)\right\}^{*} \\
=-i \int \sum \frac{d^{3} k}{2|k|} P \frac{\left|\varphi_{r}(k)\right|^{2}}{|k|+\eta}+\left.\frac{\pi}{2} \int \sum d \Omega|k|\left|\varphi_{r}(k)\right|^{2}\right|_{|k|=-\eta} .
\end{gathered}
$$

Die beiden Teile dieses Grenzwertes sind sinnvoll und somit auch die Konstanten (8) und (9). Im folgenden nennen wir die Norm der $\varphi$-Funktionen $\varkappa^{2}$, d. h.

$$
\sum_{r} N \varphi_{r}(x)=\sum_{r} N \varphi_{r}(k)=\varkappa^{2},
$$

und $x$ selbst die Koppelungskonstante. Wenn wir verlangen, daß $\gamma$ oder $\triangle \omega_{0}$ klein gegenüber $\omega_{0}$ sind, kommt das einer Bedingung für die Koppelung gleich. Wir nehmen deshalb an, daß $x \ll 1$ ist, was im Falle der Quantenelektrodynamik auch gut erfüllt ist. Für genügend kleines $\varkappa$ ist auch die Voraussetzung d) erfüllt.

Der Sprung der Funktion $\Gamma(z)$ an der negativen imaginären Achse ist infolge der Beziehung (11)

$$
S(i \eta)=2 \operatorname{Re} \lim _{\substack{\xi \rightarrow+0 \\ \eta<0}} \Gamma(\xi+i \eta)=\left.\pi \int \sum\left|\varphi_{r}(k)\right|^{2} \cdot|k| \cdot d \Omega\right|_{|k|=-\eta} .
$$

Wir wollen nun eine analytische Funktion $S(z)$ suchen, die auf der negativen jmaginären Achse mit $S(i \eta)$ übereinstimmt. Durch die An- 
wendung der Fouriertransformierten $\varphi_{r}(x)$ erhalten wir

$$
S(z)=\frac{1}{2 \pi} z \iint \sum d^{3} x^{\prime} d^{3} x \frac{\sin \left(i\left|x-x^{\prime}\right| z\right)}{z\left|x-x^{\prime}\right|} \varphi_{r}(x) \varphi_{r}^{*}\left(x^{\prime}\right) .
$$

Das Integral (13) ist sicher sinnvoll für $z=i \eta$, weil der Integrand auch endlich für $\left|x-x^{\prime}\right| \rightarrow 0$ wird. Wegen der Voraussetzung c) können wir den Ausdruck (13) beliebig oft nach $z$ im Nullpunkt differenzieren und erhalten

$$
\begin{aligned}
S^{(2 v)}(0) & =0, \\
S^{(2 v+1)}(0) & =i \frac{f^{2}}{2 \pi} \overline{\left|x-x^{\prime}\right|^{2 v}} .
\end{aligned}
$$

Die Ableitung von $S(z)$ nach $z$ hat auf der reellen Achse die rein imaginäre Form

$$
S^{\prime}(\xi)=i \frac{f^{2}}{2 \pi} \overline{\cosh \left|x-x^{\prime}\right| \xi}
$$

deren Betrag für $|\xi| \leqq \gamma$ wegen der Voraussetzung d) kleiner als $(2 \pi)^{-1}$ ist. Wegen c) ist ihr Imaginärteil in diesem Intervall positiv. $S(\xi)$ ist auch rein imaginär, und es ist

$$
\operatorname{Im} S(\xi)=\frac{f^{2}}{2 \pi} \frac{\overline{\sin h\left|x-x^{\prime}\right| \xi}}{\left|x-x^{\prime}\right|},
$$

während $0 \leqq \operatorname{Im} S(\xi)<\gamma \cdot(2 \pi)^{-1}$ für $0 \leqq \xi \leqq \gamma$ ist. Also ist

$$
0 \leqq f^{2} \frac{\overline{\sin h\left|x-x^{\prime}\right| \xi}}{\left|x-x^{\prime}\right|}<\gamma \quad \text { für } \quad 0 \leqq \xi \leqq \gamma .
$$

Diese Beziehung wird später gebraucht. Ohne eine weitere Annahme über die $\varphi$-Funktionen können wir nicht beurteilen, ob $S(z)$ überall außerhalb der imaginären Achse sinnvoll ist. Wenn jedes $\varphi_{r}(x)$ nur in einem endlichen Gebiet von Null verschieden ist, ist $S(z)$ analytisch in der ganzen Ebene. Wenn $\varphi_{r}(x)$ aber exponentiell mit $|x|$ abnimmt, ist $S(z)$ in einem Streifen um die imaginäre Achse analytisch. In allen Diskussionen um eine eventuelle analytische Fortsetzung solcher $\Gamma$-Funktionen ist in der Literatur angenommen worden, daß sich der Wert der fortgesetzten Funktion im Punkt $-i \omega_{0}$ nicht wesentlich von ihrem Wert im Weisskopf-Wigner Pol $-\gamma / 2-i \omega_{0}$ unterscheidet. Diese Annahme ist bei den gegebenen Voraussetzungen sicher durch ähnliche Überlegungen erfüllt, wie wir vorher auf der reellen Achse ausgeführt haben. Anderseits ist es bekannt, daß die Funktion $\varphi_{r}(x)$ sich in der Quantenelektrodynamik exponentiell verhält. Wir machen deshalb die Annahme, daß $S(z)$ in einem Streifen um die imaginäre Achse analytisch ist, dessen Ränder nicht parallel sein müssen. Sicher enthält dieses Gebiet den Streifen

$$
|\operatorname{Re} z| \leqq \gamma \text {. }
$$

Es ist nun klar, daß $\Gamma(z)$ über die negative imaginäre Achse im Uhrzeigersinn analytisch als $\Gamma(z)+S(z)$ fortgesetzt werden kann. Die Ableitungen der so fortgesetzten Funktion erfahren nämlich keine 
sprunghaften Änderungen an der imaginären Achse. Wir verlegen nun den Schnitt von $O$ zuerst horizontal nach links bis $-\gamma$ und dann senk-

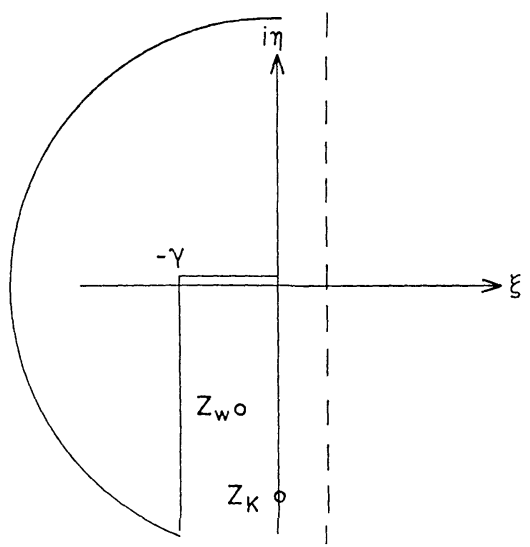

Fig. 1 recht nach unten. Die dementsprechend fortgesetzte $\Gamma$-Funktion nennen wir $A(z)$, d. h. $A=$ $\Gamma+S$ für $-\gamma<\xi<0, \eta<0$ und $A=\Gamma$ in der übrigen Ebene. Der ursprüngliche Integrationsweg in (5) und (6) (in der Figur gestrichelt) wird in der linken Halbebene durch den Bogen eines Kreises ergänzt, dessen Zentrum im Nullpunkt liegt und dessen Radius gegen $\infty$ strebt, während der Bogen in dem unteren Teil der Halbebene bis zum Schnitt führt. Dieser neue

Weg gibt keinen Beitrag. Es ist klar, daß der Integrand von (5) einen Pol in der Gegend von

$$
z_{W}=-\frac{\gamma}{2}-i\left(\omega_{0}-\triangle \omega_{0}\right)
$$

hat, während der Integrand von (6) zusätzlich den Pol $z_{K}=-i|k|$ hat. Man kann den ersten Pol genauer bestimmen, wenn der Nenner des betreffenden Integranden bis zum ersten Grad in $z-z_{W}$ entwickelt wird. Da solche Korrekturen den Charakter der Lösung nicht ändern, wollen wir sie nicht ausführen. Da nun sämtliche Singularitäten der Integranden aufgezählt sind, können wir den Integrationsweg um den Schnitt zusammenschrumpfen lassen und erhalten

1) die Residua um die Pole, $\varepsilon_{i}(t)$,

2 ) die Integrale um den Schnitt, $\sigma_{i}(t),(i=1,2)$.

An Stelle der Integrale (5) und (6) erhalten wir somit

$$
\begin{aligned}
& \psi_{1}(t)=\varepsilon_{1}(t)+\sigma_{1}(t) \\
& \psi_{2}(t)=\varepsilon_{2}(t)+\sigma_{2}(t)
\end{aligned}
$$

wobei die Residua $\varepsilon_{i}(t)$ die folgenden Formen annehmen (Siehe [15], S. 259 die Formeln (14) und (15)):

$$
\begin{aligned}
& \varepsilon_{1}(t)=e^{-i\left(\omega_{1}-\Delta \omega_{0}\right) t} \cdot e^{-\frac{\gamma}{2} t} \\
& \varepsilon_{2}(t)=-i h_{i}^{*}(1 / 2, k, r) \cdot e^{-i \omega_{2} t} \\
& \left\{\frac{e^{\left(-\frac{\gamma}{2}-i\left(\omega_{1}-\Delta \omega_{0}\right)\right) t}}{-\frac{\gamma}{2}+i\left(|k|-\left(\omega_{0}-\triangle \omega_{0}\right)\right)}+\frac{e^{-i \mid k_{i} t}}{-i|k|+i \omega_{0}+A(-i|k|)}\right\} \\
& \cong \frac{h_{i}^{*}(1 / 2, k, r)}{|k|-\left(\omega_{0}-\Delta \omega_{0}\right)+i \frac{\gamma}{2}}\left\{e^{-i\left(\omega_{2}+|k|\right) t}-e^{-i\left(\omega_{1}-\Delta \omega_{0}\right) t} \cdot e^{-\frac{\gamma}{2} t}\right\} .
\end{aligned}
$$


Die Funktionen $\varepsilon_{i}$ geben den exponentiellen Charakter der Lösung wieder. Die Integrale um den Schnitt $\sigma_{i}$ werden einen weiteren, aber unbedeutenden Beitrag zum Exponentialzerfall geben. Dazu geben sie einen Beitrag, der nicht exponentiell ist und für sehr große Zeiten für die Gesamtlösung wesentlich wird.

\section{\$3. Auswertung der Integrale um den Schnitt}

Im folgenden betrachten wir zuerst die zerfallende Amplitude $\psi_{1}(t)$ und berechnen das Integral um den Schnitt $\sigma_{1}(t)$. Wenn wir den Integrationsweg um den Schnitt mit $U$ bezeichnen, ist

$$
\sigma_{1}(t)=\frac{1}{2 \pi i} \int_{U} e^{z t}\left(z+i \omega_{0}+A\right)^{-1} d z
$$

Da $\varepsilon_{1}(t)$ im Grenzwert für $t \rightarrow 0$ den richtigen Anfangswert 1 ergibt, ist es nicht zu erwarten, daß $\sigma_{t}(t)$ für sehr kleine Zeiten einen interessanten Beitrag gibt. Wir wollen deshalb versuchen für $t \neq 0$ eine Aufteilung des Integrals in die Summe

$$
\sigma_{1}(t)=\tau_{1}(t)+\zeta_{1}(t)
$$

derart anzugeben, daß der eine Teil $\tau_{1}(t)$ für große Zeiten viel bedeutender wird als der zweite, $\zeta_{1}(t)$. Dabei sollte die Form von $\tau_{1}(t)$ möglichst genau ermittelt werden.

Den Integrationsweg um den waagerechten Teil des Schnittes, d. h. um $-\gamma \leqq \xi \leqq 0$, im positiven Umlaufsinn bezeichnen wir mit $W$. Der erste Schritt zur Auswertung von $\sigma_{1}$ ist, das Integral $\mathscr{I}_{W}$ längs des Weges $W$ auszurechnen:

$$
\mathscr{I}_{W}(t)=\frac{1}{2 \pi i} \int_{W} e^{z t}\left(z+i \omega_{0}+A\right)^{-1} d z .
$$

Dazu machen wir von der folgenden algebraischen Umformung Gebrauch,

$$
\frac{1}{z+i \omega_{0}+A}=\frac{1}{z+i \omega_{0}}-\frac{A}{\left(z+i \omega_{0}\right)\left(z+i \omega_{0}+A\right)}
$$

die wir durch Iteration auf die Form

$$
\frac{1}{z+i \omega_{0}+A}=\frac{1}{z+i \omega_{0}}-\frac{A}{\left(z+i \omega_{0}\right)^{2}}+\frac{A^{2}}{\left(z+i \omega_{0}\right)^{2}\left(z+i \omega_{0}+A\right)},
$$

bringen. Dieser Ausdruck ist die Teilsumme einer geometrischen Reihe mit Restglied. Eingesetzt in (18) hat er eine Summe von Integralen zur Folge, wovon das erste verschwindet, wenn der Integrationsweg an die Ufer des Schnittes verlegt wird. Wir erhalten also

$$
\begin{aligned}
\mathscr{I}_{W}(t) & =\mathscr{I}_{1}(t)+\mathscr{R}_{1}(t) \\
\mathscr{I}_{1}(t) & =\frac{1}{2} \frac{1}{\pi i} e^{-i \omega_{2} t} \int_{0}^{\gamma} e^{-\xi t} \frac{S(\xi)}{\left(-\xi+i \omega_{0}\right)^{2}} d \xi \\
\cdot \mathscr{R}_{1}(t) & =\frac{1}{2} \frac{1}{\pi i} e^{-i \omega_{2} t} \int_{W} e^{-z t} \frac{A^{2}(z)}{\left(z+i \omega_{0}\right)^{2}\left(z+i \omega_{0}+A\right)} d z
\end{aligned}
$$




\section{A. Berechnung des Integrals $\mathscr{I}_{1}(t)$}

Setzt man das Integral (13) in $\mathscr{I}_{1}(t)$ nach (19) ein, und vertauscht man die Reihenfolge der Integrationen, so erhält man

$$
\begin{aligned}
\mathscr{I}_{1}(t) & =\frac{1}{8 \pi^{2}} e^{-i \omega_{2} t} \iint \sum_{r} d^{3} x d^{3} x^{\prime} \varphi_{r}(x) \varphi_{r}^{*}\left(x^{\prime}\right) i_{1}(\varrho) \\
i_{1}(\varrho) & =\int_{0}^{\gamma} d \xi \frac{e^{-\xi t}\left(e^{\varrho \xi}-e^{-\varrho \xi}\right)}{\varrho\left(-\xi+i \omega_{0}\right)^{2}} \\
\varrho & =\left|x-x^{\prime}\right| .
\end{aligned}
$$

Die Vertauschung der Integrationen ist erlaubt, weil das Integral (13) gleichmäßig in $z$ konvergiert. Das innere Integral $i_{1}(\varrho)$ wollen wir wieder in einen wesentlichen Bestandteil und Rest aufteilen:

$$
\begin{aligned}
i_{1}(\varrho) & =-\frac{1}{\omega_{0}^{2}} h(\varrho)+\frac{1}{\omega_{0}^{2}} h(\varrho) \\
h(\varrho) & =\int_{0}^{\gamma} d \xi \frac{e^{-\xi t}\left(e^{\varrho \xi}-e^{-\varrho \xi}\right)}{\varrho} \\
\cdot r(\varrho) & =\int_{0}^{\gamma} d \xi \frac{\xi\left(\xi-2 i \omega_{0}\right)}{\left(-\xi+i \omega_{0}\right)^{2}} \frac{e^{-\xi t}\left(e^{\varrho \xi}-e^{-\varrho \xi}\right)}{\varrho} .
\end{aligned}
$$

Das Integral $h(\varrho)$ können wir sicher auswerten, und wir erhalten, wenn $t \neq \varrho$ ist

$$
h(\varrho)=\frac{2}{t^{2}-\varrho^{2}}-e^{-\gamma t} \frac{e^{\gamma \varrho}(t+\varrho)-e^{-\gamma \varrho(t-\varrho)}}{\varrho\left(t^{2}-\varrho^{2}\right)} .
$$

In dieser Form darf $\varrho$ gegen Null gehen. Entwickelt man $e^{\gamma e}$ und $e^{-\gamma e}$ in Potenzreihen, kann man $\varrho$ in Zähler und Nenner des zweiten Bruchs wegkürzen. Den ersten Faktor im Integranden von $\iota(\varrho)$ können wir als eine schnell konvergierende Potenzreihe in $\xi / \omega_{0}$ darstellen. Da sämtliche zeitliche Ableitungen von $h(\varrho)$ existieren, dürfen wir dann schreiben

$$
\iota(\varrho)=-\frac{2 i}{\omega_{0}} \frac{\partial}{\partial t} h(\varrho)+\frac{3}{\omega_{0}^{2}} \frac{\partial^{2}}{d t^{2}} h(\varrho)+\frac{2 i}{\omega_{0}^{3}} \frac{\partial^{3}}{d t^{3}} h(\varrho)+\cdots,
$$

und die Koeffizienten vor den höheren Ableitungen werden alle die Beträge $2 / \omega_{0}^{v}$ haben, wenn $v$ der Grad der Ableitung ist.

Die äußere Integration in (20) ist nun eine Mittelung nach der Art, die im vorigen Paragraphen erklärt wurde. Wir bekommen also

$$
\mathscr{I}_{1}(t)=\frac{f^{2}}{8 \pi^{2} \omega_{0}^{2}} e^{-i \omega_{2} t}\{-\overline{h(\varrho)}+\overline{r(\varrho)}\}
$$

und es fragt sich, ob die Formen (22) und (23) für die Mittelung angewandt werden dürfen. Eine Schwierigkeit entsteht bei $\varrho=t$, da beide Glieder des Ausdrucks (22) dann singulär werden. Diese Schwierigkeit wird gehoben, indem wir bei der Integration nach $\varrho$ den Hauptwert bilden. Die Umgebung von $\varrho=t$ gibt dann nur einen endlichen Beitrag zum Integral des ersten und ebenso des zweiten Gliedes. Diese Beiträge sind überdies für $t \gg 1$ klein, da das Produkt $\varphi_{r}(x) \varphi_{r}^{x}\left(x^{\prime}\right)$ für große $\varrho$ 
sehr kleinen Betrag hat. Bei der Mittelung über die einzelnen Glieder der Reihe (23) können wir die Reihenfolge der Integrationen wieder vertauschen

$$
\overline{\frac{\partial^{v}}{\partial t^{v}} h(\varrho)}=(-1)^{v} \int_{0}^{\gamma} d \xi \cdot \xi^{v} \frac{\overline{e^{-\xi t}\left(e^{\varrho \xi}-e^{-0 \xi}\right)}}{\varrho} .
$$

Wenn wir die Beziehung (14) berücksichtigen, ist es klar, daß die Mittelung über das erste Glied von (22) ein größeres Gewicht als die Mittelung über das zweite Glied erhält, wenn $t>1 / \gamma$ wird. Wenn $t$ etwa $10 \mathrm{mal}$ so groß geworden ist, fällt das zweite Glied der Mittelung gar nicht mehr in Betracht. Ebenfalls aus (14) folgt, daß der Ausdruck (25) so abgeschätzt werden darf:

$$
\overline{\frac{\partial^{\nu}}{\partial t^{v}} h(\varrho)}<\gamma^{v} h(\varrho) \text {. }
$$

Wegen (14) ist $\overline{h(\varrho)}$ positiv. Wir können dann auch $\overline{r(\varrho)}$ wie folgt abschätzen

$$
\overline{|r(\varrho)|}<3 \overline{h(\varrho)}\left(\frac{\gamma}{\omega_{0}}+\left(\frac{\gamma}{\omega_{0}}\right)^{2}+\cdots\right)=3 \frac{\gamma}{\omega_{0}} \overline{h(\varrho)} \frac{1}{1-\gamma / \omega_{0}} .
$$

Nun ist $\frac{\gamma}{\omega_{0}} \ll 1$. Das Restglied der Aufteilung (24) ist deshalb im Betrag verschwindend klein gegenüber dem Hauptglied und zwar für alle Zeiten. Wenn wir auch die Form (23) berücksichtigen, ist es dazu klar, daß $\overline{\iota(\varrho)}$ viel schneller für große $t$ verschwindet als $\overline{h(\varrho)}$. Damit dürfen wir folgendes feststellen: Wenn wir anstatt der Beziehung (24)

$$
\mathscr{I}_{1}(t) \cong-\frac{f^{2}}{4 \pi^{2} \omega_{0}^{2}} e^{-i \omega_{2} t} \frac{1}{t^{2}-\varrho^{2}}
$$

schreiben, ist das für $t>1 / \gamma$ eine gute approximative und für $t \rightarrow \infty$ die exakte asymptotische Lösung für das Integral (20).

\section{B. Abschätzung des Restgliedes $\mathscr{R}_{1}(t)$}

Das Restglied $\mathscr{R}_{1}(t)$ nach (19) kann in der Form

$$
\mathscr{R}_{1}(t)=\frac{1}{2 \pi i} e^{-i \omega_{2} t} \int_{0}^{\gamma} e^{-\xi t} \frac{S(\xi)}{\left(-\xi+i \omega_{0}\right)^{2}} \cdot j(\xi) d \xi
$$

geschrieben werden, und die Funktion $j(\xi)$ hängt dann von der $\Gamma$-Funktion ab, die singulär im Nullpunkt ist. Trotzdem existiert der Grenzwert von $j(\xi)$, wenn $\xi \rightarrow 0$ strebt. Bis auf noch kleinere Größen ist dieser Grenzwert vom Betrag $|j(0)|=2 \frac{|\Gamma(0)|}{\omega_{0}} \ll 1$. Die $\Gamma$-Funktion kann in der Gegend des Nullpunktes auf der reellen Achse am besten abgeschätzt werden, wenn das Integral (7) zuerst über eine kleine Kugel $|k| \leqq u$ und dann über das Außengebiet berechnet wird. Dann ist

$$
|\Gamma(-\xi)-\Gamma(0)|<\frac{\xi}{u}|\Gamma(0)|
$$


bis auf Fehler von der Größenordnung $\frac{1}{2} u$. Durch passende Wahl von $u$ können wir beweisen, daß $|j(\xi)|$ auf der ganzen Strecke $0 \leqq \xi \leqq \gamma$ von der gleichen Größenordnung wie in 0 ist. Wenn wir (28) mit $\mathscr{I}_{1}(t)$ nach (19) vergleichen, ist es deshalb klar, daß $\mathscr{R}_{1}(t)$ nur eine äußerst geringfügige Korrektur in $\mathscr{I}_{W}(t)$ gibt, wenn diese Restfunktion zu $\mathscr{I}_{1}(t)$ addiert wird. Mit etwas mehr Mühe können wir beweisen, daß $\mathscr{R}_{1}(t)$ gegenüber $\mathscr{I}_{1}(t)$ für $t \rightarrow \infty$ asymptotisch zu vernachlässigen ist.

\section{Auswertung des Integrals $\sigma_{1}(t)$}

Wir schreiben jetzt

$$
\tau_{1}(t)=-\frac{f^{2}}{4 \pi^{2} \omega_{0}^{2}} e^{-i \omega_{2} t \overline{\frac{1}{t^{2}-\varrho^{2}}}}
$$

und behaupten, daß dieser Ausdruck die ursprünglich in diesem Abschnitt gesuchte asymptotische Lösung des Integrals um den Schnitt ist. Wir haben noch nicht das Integral um den senkrechten Teil des Schnittes diskutiert. Nennen wir dieses Integral $\mathscr{I}_{S}$. Es ist dann

$$
\begin{aligned}
\mathscr{I}_{S}(t)= & e^{-\gamma t} \frac{1}{2 \pi} e^{-i \omega_{2} t} \\
& \cdot\left\{\int_{-\infty}^{0} e^{i \eta t} \frac{1}{-\gamma+i \omega_{0}+i \eta+\Gamma(-\gamma+i \eta)+S(-\gamma+i \eta)} d \eta\right. \\
& \left.-\int_{-\infty}^{0} e^{i \eta t} \frac{1}{-\gamma+i \omega_{0}+i \eta+\Gamma(-\gamma+i \eta)} d \eta\right\} .
\end{aligned}
$$

Der Betrag des Klammerausdrucks ist sicher endlich, und wenn $\omega_{0} \gg \mathbf{1}$ ist, kann er als $\ll 1$ geschätzt werden. Weil der Exponent $-\gamma t$ das doppelte des Exponenten in der Lösung $\varepsilon_{1}(t)$ ist, stellt $\mathscr{I}_{S}$ keine wesentliche Korrektur des Exponentialzerfalls dar.

Damit ist nun bewiesen worden, daß die Funktion $\tau_{1}(t)$ nach (29) die asymptotische Form des Integrals $\sigma_{1}(t)$ in bezug auf die Zeit darstellt. Die Funktion ist für $t>1 / \gamma$ auch eine sehr gute approximative Lösung von $\sigma_{1}(t)$. Sie ist dann für große Zeiten die gesuchte Abweichung von dem Exponentialzerfall $\varepsilon_{1}(t)$. Der Vergleich der Funktion $\varepsilon_{1}(t)$ nach (16) mit $\tau_{1}(t)$ nach (29), zeigt uns, für welche Zeiten

$$
t>t_{e}
$$

$\left|\varepsilon_{1}(t)\right|$ vergleichbar mit oder kleiner als $\left|\tau_{1}(t)\right|$ ist. Wir definieren

$$
\alpha=\ln \frac{8 \sqrt{2} \pi \omega_{0}}{\gamma f}
$$

und erhalten als gute Darstellung der extremen Zeit $t_{\boldsymbol{e}}$

$$
t_{e}=\frac{4}{\gamma}\{\alpha+\ln \alpha\} \text {. }
$$


Wenn die Zerfallszeit diesen extremen Wert übertrifft, verliert $\psi_{1}(t)$ ganz den exponentiellen Zerfallscharakter und die Form $\tau_{1}(t)$ überwiegt.

\section{Auswertung des Integrals $\sigma_{2}(t)$}

Die Amplitude des zerfallenen Zustandes $\psi_{2}(t)$ hat als Hauptteil das Residuum $\varepsilon_{2}(t)$, das in (16) angegeben ist. Die exakte Form des Residuums hat für $t=0$ nicht genau den richtigen Anfangswert 0 . Daraus kann man schließen, daß die Auswertung des Integrals $\sigma_{2}(t)$ schon für $t=0$ eine sinnvolle Größe gibt. Im folgenden rechnen wir jedoch mit der leicht modifizierten Schlußform von $\varepsilon_{2}(t)$, die ebenfalls in (16) angegeben wird und den richtigen Anfangswert 0 hat. Damit wird $\sigma_{2}(t)$ wieder nur für $t \neq 0$ interessant. Die Auswertung und Abschätzung des Integrals $\sigma_{2}(t)$ kann genau so ausgeführt werden, wie es vorher für $\sigma_{1}(t)$ getan wurde. Nur tritt hier die Schwierigkeit auf, daß der Integrand für kleine $|k|$ einen Pol sehr nahe dem Verzweigungspunkt hat. Eine genaue Analyse eines derartigen Problems hat B. L. VAN DER WAERDEN [20] in seiner Darstellung der Sattelpunktsmethode gegeben, indem er ein Glied mit dem betreffenden Pol als einzige singuläre Stelle von dem Integranden trennt. Es ist klar, daß $\sigma_{2}(t)$ für $t \rightarrow \infty$ verschwindet. Damit ist der Grenzwert von $\psi_{2}(t)$ für $t \rightarrow \infty$ dergleiche wie derjenige des Residuums $\varepsilon_{2}(t)$. Der Betrag dieses Grenzwertes

$$
\frac{\left|h_{2}(1 / 2, k, r)\right|}{|| k\left|-\left(\omega_{0}-\Delta \omega_{0}\right)+i \frac{\gamma}{2}\right|}
$$

ist physikalisch das interessanteste an der Amplitude $\psi_{2}(t)$. Wir werden somit nicht die genaue Art der Abweichung $\sigma_{2}(t)$ für kleinere Zeiten hier auswerten. Durch einen Kunstgriff, der ebenfalls aus der eben erwähnten Arbeit VAN DER WAERDENs stammt, wollen wir abor eine einfache Integralform angeben, die für genügend hohe Zeiten der Funktion $\sigma_{2}(t)$ beliebig genau entspricht: Als wesentlicher Teil des Integrals $\sigma_{2}(t)$ interessiert uns die Integration um den waagerechten Teil des Schnittes. Wir nennen ihn $\mathscr{J}_{W}$ und es ist

$$
\mathscr{J}_{W}=\frac{1}{2 \pi i} e^{-i \omega_{2} t} \int_{W} e^{z t} \frac{\left(i h_{i}(1 / 2, k, r)\right)^{*}}{(z+i|k|)\left(z+i \omega_{0}+A(z)\right)} d z .
$$

Es kann dann die folgende Beziehung zwischen $\mathscr{J}_{W}$ nach $(31)$ und $\mathscr{I}_{W}$ nach (18) gebildet werden:

$$
\frac{d}{d t}\left\{e^{i\left\{|k|+\omega_{2}\right\} t} \mathscr{J}_{W}\right\}=\left(i h_{i}(1 / 2, k, r)^{*} e^{i \omega_{2} t} \mathscr{I}_{W} .\right.
$$

Mit der oben angegebenen Auskunft über das Verhalten der Integrale um den Schnitt im Unendlichen können wir die Gl. (32) integrieren. Wenn $t$ groß genug ist, bekommen wir

$$
\mathscr{J}_{W}=i e^{-i\left\{|k|+\omega_{2}\right\} t} h_{i}^{*}(1 / 2, k, r) \int_{t}^{\infty} e^{i \omega_{2} t^{\prime}} \tau\left(t^{\prime}\right) d t^{\prime} .
$$


Wenn wir $\tau(t)$ nach (29) in (33) einsetzen, erhalten wir wieder einen Ausdruck in negativen Potenzen von $t$.

Anmerkungen. Die Koppelung des betrachteten Systems wird als der Betrag $\left\|\varphi_{r}(x)\right\|$ definiert und $\varkappa$ genannt. Andererseits wird das Integral $\iint \Sigma d^{3} x d^{3} x^{\prime} \varphi_{r}(x) \varphi_{r}^{*}\left(x^{\prime}\right)$ gleich $f^{2}$ gesetzt. Wenn wir die Wechselwirkung im Rahmen der Quantenelektrodynamik [2] formulieren, wird $f$ gleich $\varkappa$. Weil aber die Herleitung der Grundformen der Quantentheorie aus der Lagrangeform von vielen Physikern bestritten wird, wird hier zwischen $x$ und $f$ unterscheiden.

Die Form der Integrale (5) und (6) dürfte allgemein als ein gutes Feldmodell gelten. Wenn die Integrale auf den Zerfall von Teilchen mit Ruhemasse $\mu \neq 0$ angewandt werden, muß $|k|$ in (5), (6) und (7) durch $\omega_{k}=\sqrt{k^{2}+\mu^{2}}$ ersetzt werden. Die schon beschriebene Methode zur Auswertung der formalen Lösung wird dadurch nicht geändert.

\section{Literatur}

1. von Neumann, J.: Mathematical foundations of quantum mechanics. Princeton: Princeton University Press 1955.

2. Heitler, W.: The quantum theory of radiation, Chap. 4 and 5. Oxford: University Press 1954.

3. Weisskopf, V., u. E. Wigner: Z. Physik 63, 54 (1930); 65, 18 (1930).

4. Heitler, W., and S. T. MA: Proc. Roy. Irish Acad. 52, 109 (1949).

5. Arnous, E., u. S. Zienau: Helv. Phys. Acta 24, 279 (1951).

6. 一, u. K. Bleuler: Helv. Phys. Acta 25, 581 (1952).

7. - Helv. Phys. Acta 25, 631 (1952).

8. 一, and W. Heitler: Proc. Roy. Soc. Lond. A 220, 290 (1953).

9. Matthews, P. T., and Abdus Salam: Phys. Rev. 112, 283 (1958); 115, 1979 (1959).

10. HöHLER, E.: Z. Physik 152, 546 (1958).

11. Hellund, B. J.: Phys. Rev. 89, 919 (1953).

12 KälléN, A. O. G.: Handbuch der Physik, Band 5, 1, Kap. V, § 28. BerlinGöttingen-Heidelberg: Springer 1958.

13. Messtah, A.: Quantum mechanics, Chap. 21, § 13. Amsterdam: North-Holland Publ. Comp. 1962.

14. Friedrich, Marianne: Commun. Math. Phys. 2, 327 (1966).

15. Ingólfssson, Ketill: Helv. Phys. Acta 40, 237 (1967).

16. Fock, V.: Konfigurationsraum und zweite Quantelung. Z. Physik 75, 622 (1932).

17. Schwartz, L.: Théorie des Distributions. Paris: Hermann 1950.

18. Friedrichs, K.: Mathematicals aspects of the quantum theory of fields. New York: Interscience 1953.

19. van Der Waerden, B. L.: Helv. Phys. Acta 36, 7 (1963).

20. - Appl. Sci. Res. B 2, 33 (1950).

K. Ingólfasson

Department of Mathematics

University of Arizona

Tucson, Arizona 85721

USA 